\title{
Avian influenza virus in water: global subtype diversity and spatial distribution patterns
}

\author{
Jessica Mateus-Anzola ${ }^{1}$, Beatriz Martínez-López ${ }^{2}$, Ana Cecilia Espinosa-García ${ }^{3}$, and \\ Rafael Ojeda-Flores ${ }^{1}$ \\ ${ }^{1}$ Universidad Nacional Autonoma de Mexico Facultad de Medicina Veterinaria y Zootecnia \\ ${ }^{2}$ University of California Davis Department of Medicine and Epidemiology \\ ${ }^{3}$ Universidad Nacional Autonoma de Mexico Instituto de Ecologia
}

May 7, 2021

\begin{abstract}
The current COVID-19 pandemic highlights the need for zoonotic infectious disease surveillance. Avian influenza virus (AIV) poses a significant threat to animal and public health due to its pandemic potential. Virus-contaminated water has been suggested as an important AIV spread mechanism among multiple species. Nevertheless, few studies have characterized the global AIV subtype diversity and distribution in environmental water. Therefore, this study aims to provide an updated descriptive and phylogenetic analysis of AIVs isolated in water samples from high risk-sites for influenza outbreaks (i.e., live bird markets, poultry farms, and wild bird habitats) on a global scale. A total of 234 hemagglutinin (HA) gene sequences of 21 subtypes were reported from nine countries between 2003 - 2020. Fourteen AIV subtypes were solely reported from Asian countries. Most of the viral sequences were obtained in China and Bangladesh with $47.44 \%$ and $23.93 \%$, respectively. Likewise, the greatest global AIV subtype diversity was observed in China with twelve subtypes. Live bird markets represented the main sampling site for AIV detection in water samples (64.10\%), mostly from poultry cage water. Nevertheless, the highest subtype diversity was observed in water samples from wild bird habitats, especially from the Izumi plain and the Dongting Lake located in Japan and China, respectively. Water from drinking poultry troughs evidenced the greatest subtype diversity in live bird markets, meanwhile, environmental water used by ducks had the highest number of different subtypes in poultry farms. The maximum-likelihood phylogenetic tree showed that some HA sequences were closely related among different poultry/wild bird-related environments from different geographic origins. Therefore, the results suggest that even though the availability of HA gene sequences in public-access databases varies greatly among countries, environmental AIV surveillance represents a useful tool to elucidate potential viral diversity in wild and domestic bird populations.
\end{abstract}

\section{Avian influenza virus in water: global subtype diversity and spatial distribution patterns}

Jessica Mateus-Anzola a , Beatriz Martínez-López ${ }^{\text {, }}$, Ana Cecilia Espinosa-García c ${ }^{\text {, Rafael Ojeda-Flores a* }}$

a Laboratorio de Ecología de Enfermedades y Una Salud, Departamento de Etología, Fauna Silvestre y Animales de Laboratorio, Facultad de Medicina Veterinaria y Zootecnia, Universidad Nacional Autónoma de México, 04510, Mexico.

b Center for Animal Disease Modeling and Surveillance (CADMS), Department of Medicine \& Epidemiology, School of Veterinary Medicine, University of California-Davis, Davis, CA, 95616, United States.

c Laboratorio Nacional de Ciencias de la Sostenibilidad, Instituto de Ecología, Universidad Nacional Autónoma de México, 04510, Mexico.

*Corresponding author: Rafael Ojeda-Flores. Laboratorio de Ecologia de Enfermedades y Una Salud, Departamento de Etologia, Fauna Silvestre y Animales de Laboratorio, Facultad de Medicina Veterinaria 
y Zootecnia, Universidad Nacional Autonoma de Mexico, Av. Universidad 3000, Edificio A, Delegacion Coyoacan, Col. Ciudad Universitaria, 04510, Mexico city, Mexico. Tel.: +52 5513207039.

E-mail address : ojedar@unam.mx

\section{Summary}

The current COVID-19 pandemic highlights the need for zoonotic infectious disease surveillance. Avian influenza virus (AIV) poses a significant threat to animal and public health due to its pandemic potential. Virus-contaminated water has been suggested as an important AIV spread mechanism among multiple species. Nevertheless, few studies have characterized the global AIV subtype diversity and distribution in environmental water. Therefore, this study aims to provide an updated descriptive and phylogenetic analysis of AIVs isolated in water samples from high risk-sites for influenza outbreaks (i.e., live bird markets, poultry farms, and wild bird habitats) on a global scale. A total of 234 hemagglutinin (HA) gene sequences of 21 subtypes were reported from nine countries between 2003 - 2020. Fourteen AIV subtypes were solely reported from Asian countries. Most of the viral sequences were obtained in China and Bangladesh with $47.44 \%$ and $23.93 \%$, respectively. Likewise, the greatest global AIV subtype diversity was observed in China with twelve subtypes. Live bird markets represented the main sampling site for AIV detection in water samples (64.10\%), mostly from poultry cage water. Nevertheless, the highest subtype diversity was observed in water samples from wild bird habitats, especially from the Izumi plain and the Dongting Lake located in Japan and China, respectively. Water from drinking poultry troughs evidenced the greatest subtype diversity in live bird markets, meanwhile, environmental water used by ducks had the highest number of different subtypes in poultry farms. The maximum-likelihood phylogenetic tree showed that some HA sequences were closely related among different poultry/wild bird-related environments from different geographic origins. Therefore, the results suggest that even though the availability of HA gene sequences in public-access databases varies greatly among countries, environmental AIV surveillance represents a useful tool to elucidate potential viral diversity in wild and domestic bird populations.

Keywords: Environmental Monitoring; Epidemiology; Hemagglutinin; Infectious disease; Phylogenetic Analysis; Water Microbiology.

\section{Introduction}

Waterborne transmission is considered a significant route for avian influenza viruses (AIV) dissemination (Lebarbenchon et al., 2011). This indirect route of transmission is known as one of the main determinants of disease dynamics, since water can be simultaneously contaminated by different viral strains and infect multiple animals, including wild birds (Numberger et al., 2019; Roche et al., 2009).

Waterfowl are recognized as the natural reservoir of IAV and play a key role in the viral spread and diversity (Montalvo-Corral \& Hernandez, 2010). Infected wild ducks shed feces with high AIV concentrations into aquatic environments (Dovas et al., 2010). Viruses shedding into the water have the potential to infect any other waterfowl within bird populations by drinking or filtering the virus-contaminated water (Keeler et al., 2014). Therefore, viral transmission among wild aquatic birds mainly occurs via the fecal-oral route through contaminated water (Brown et al., 2007).

Environmental water where resident and migratory waterfowl congregate has a significant role in the AIV ecology and epidemiology (Numberger et al., 2019). Viruses shed before migration can persist in the water over winter and become a source of infection to birds returning during spring (Brown et al., 2007). This environmental persistence may enable short- and long-term maintenance of AIV among spatially or temporally separated wild bird populations (Lickfett et al., 2018).

Wild bird habitats close to livestock production systems also represent high-risk zones for AIV interspecies transmission (Achenbach \& Bowen, 2011; Harris et al., 2017). Barnyard fowls from backyard farms can use open waters and become indirectly infected by wild waterfowls congregated in the same habitats (Cappelle et al., 2011). Likewise, other farm animals, such as pigs, can be AIV infected by contaminated surface water 
collected from natural or artificial ponds and used for cleaning barns and watering animals (Karasin et al., 2000; Ma et al., 2007).

In addition to the wild duck-livestock animal interface, live bird markets constitute high-risk sites for harboring and spreading AIV (Khan et al., 2018). Wet markets promote close contact between different animal species which facilitates the genetic reassortment of influenza viruses (Vergne et al., 2019). Wild and domestic birds (including infected birds) use to share cages, feed, and water trays. Therefore, birds from live bird markets can be co-infected with different AIV subtypes (Khan et al., 2018).

Globally, multiple subtypes of low- and high-pathogenic avian influenza (LPAI and HPAI) have been identified based on epidemiological surveillance (Machalaba et al., 2015). Environmental AIV surveillance is considered a convenient, noninvasive, and sensitive method for conducting AIV detection (Leung et al., 2007). Avian influenza surveillance in poultry-related environments, including live poultry markets, wild bird habitats, slaughterhouses, and poultry farms is conducted annually in some countries (Zhang et al., 2019). However, the global diversity and distribution of AIVs in environmental water widely used by waterfowl and poultry have been scarcely studied. In this study, we aim to address this knowledge gap by characterizing the AIV diversity and its spatial distribution in water samples from areas considered to be at high risk for avian influenza outbreaks (i.e., wild bird habitats, live bird markets, poultry farms) on a global scale.

\section{Materials and Methods}

\section{Data collection from open-access databases}

A total of 2920 AIV hemagglutinin (HA) gene sequences were collected until 04 February 2021 using the keywords: "environment", "water", "sewage", "wastewater", "surface_water", and "drinking_water" from the NCBI Influenza Virus Database (https://www.ncbi.nlm.nih.gov/genomes/FLU/Database/nphselect.cgi), the GISAID EpiFlu Influenza database (https://www.gisaid.org/), the Influenza Research Database (IRD,https://www.fludb.org/) and the OpenFlu database (http://openflu.vital-it.ch/). All nonwater sample sequences detected in animal environments and incomplete subtype data were removed. The resulting dataset was de-replicated to remove identical records based on the Accession Numbers (Table S1). The remaining $234 \mathrm{HA}$ gene sequences compose the final dataset used for the data analysis.

\section{Data analysis}

Data from subtype, country, sampling site, and water source were extracted and analyzed. Sample sites were live bird market (LBM), poultry farm (PF), wild bird habitat (WBH), or unknown (UNK). Water sources were drinking water trough $(\mathrm{DW})$, poultry cage water $(\mathrm{PCW})$, sewage $(\mathrm{SW})$, turkey barn water $(\mathrm{TBW})$, hand-wash water (HWW), carcass-wash water (CWW), duck's environmental water (DEW), or unknown (UNK). The category "unknown" referred to not clearly stated information. Data analysis was conducted using graphs and maps created in RStudio using the ggplot2 package (Wickham, 2016) and Mapcharnet(r) (https://mapchart.net).

\section{Phylogenetic analysis}

From the 234 sequences dataset, 26 HA partial or identical sequences were removed. A total of 208 complete HA gene sequences were aligned using the MUSCLE algorithm (Edgar, 2004). A circular phylogenetic tree was constructed by the Maximum-likelihood method with 1000 bootstrap replicates using the best-fit nucleotide substitution model in MEGA X (Kumar et al., 2018) and was edited with FigTree v1.4.4 software (Rambaut, 2018). The nucleotide substitution model was selected using the Akaike and Bayesian information criteria (AIC and BIC) (Kumar et al., 2018).

\section{Results}

A total of $234 \mathrm{HA}$ gene sequences of AIVs gathered in water samples were reported from nine countries between 2003 - 2020. No AIV sequences were reported from Africa, Oceania, or South America. Most of the AIV sequences were obtained from China $(47.44 \%, \mathrm{n}=111)$ and Bangladesh $(23.93 \%, \mathrm{n}=56)$, mainly from 
two subtypes: LPAI H9N2 and HPAI H5N1. The H10N7 was the only subtype described in Europe. Most subtypes (H3N2, H4N8, H4N9, H5N1, H5N6, H5N8, H6N2, H6N6, H7N9, H9N2, H10N8, H11N2, H11N9, and H12N7) were reported only by Asian countries.

The highest AIV diversity was observed in China with twelve subtypes followed by Japan with six subtypes. H3N8 and H4N6 subtypes were described in both Asian and American countries. H4N9, H7N9, H10N8, H11N2, and H12N7 subtypes were exclusively reported in China. Similarly, H4N2, H4N8, and H6N6 subtypes were solely detected in Mexico, Japan, and Vietnam, respectively. Likewise, H1N1, H5N2, and H7N2 subtypes were only isolated in the United States (Figure 1).

The AIV sequences were detected in water samples from live bird markets $(64.10 \%, \mathrm{n}=150)$, wild bird habitats $(19.23 \%, \mathrm{n}=45)$, poultry farms $(12.82 \%, \mathrm{n}=30)$, and unknown sampling site $(3.85 \%, \mathrm{n}=9)$. AIV sequences from live bird markets were mainly reported by Asian countries (98.67\%, $\mathrm{n}=148)$, especially China $(54.66 \%, \mathrm{n}=82)$ and Bangladesh $(37.33 \%, \mathrm{n}=56)$. The United States was the only country on the American continent that reported AIV sequences from a live bird market $(1.33 \%, \mathrm{n}=2)$ (Figure 2A).

Likewise, AIV sequences from wild bird habitats and poultry farms were detected in both Asia and America. Most of the AIV sequences from wild bird habitats were reported in China $(46.67 \%, \mathrm{n}=21)$ and Japan $(35.56 \%, \mathrm{n}=16)$ (Figure 2B), especially from the Dongting Lake (Hunan) and the Izumi Plain (Kagoshima), respectively. However, IAV sequences were also identified in water samples from the Qinghai lake in China and from a Kodai pond in a Japanese zoo. In the American continent, the IAV sequences were reported in Mexico, the United States, and Canada (Figure 2B). In particular, IAV sequences were detected from the Agassiz National Wildlife Refuge and the Thief Lake Wildlife Management Area in Minnesota, United States, filtered wetland water in Alaska, United States, a wetland in Central Mexico, and a wild bird habitat in Alberta, Canada.

The IAV sequences from poultry farms were mainly reported in the United States $(66.67 \%$, n=20) (Figure $2 \mathrm{C}$ ), corresponding to water samples from barns of turkey flocks in Minnesota and Nebraska. One IAV sequence belonged to a free-range farm in the Netherlands. All the IAV sequences (H3N2, H3N8, H4N9, H11N2, and H12N7) detected from poultry farms in China $(20 \%, n=6)$ were located close to aquatic environments (i.e., a domestic duck and goose farm close to a river in Hunan; as well as duck farms in the Dongting Lake region, China). On the other hand, nine AIV sequences (H5N1, H5N6, H6N6, H7N9, and H11N9) from four Asian countries did not specify the sampling site (Figure 2D).

The highest subtype diversity was found in water samples from wild bird habitats with eleven subtypes (H1N1, H3N8, H4N2, H4N6, H4N8, H5N1, H5H6, H5N8, H6N2, H9N2, and H10N8). The wild bird habitats in Japan represented the highest subtype diversity with six subtypes (H3N8, H4N6, H4N8, H5N6, H5N8, and H6N2), two of them have also been reported from North American wild bird habitats (H3N8 and H4N6) (Figure 2B).

In the context of live bird markets, seven subtypes (H3N2, H5N1, H5N6, H6N2, H7N9, H9N2, and H11N9) were reported in Southeast Asia, and one subtype (H7N2) was detected in the United States (Figure 2A). Similarly, in poultry farms, six subtypes (H3N2, H3N8, H4N9, H5N1, H11N2, and H12N7) were reported in Southeast Asia, one subtype (H10N7) in the Netherlands, and one subtype (H5N2) in the United States (Figure 2C). China evidenced the highest subtype diversity in both live bird markets and poultry farms.

The live bird market samples were obtained from poultry cage water $(27.33 \%, \mathrm{n}=41)$, sewage $(21.33 \%$, $\mathrm{n}=32)$, and contaminated drinking water troughs $(14.67 \%, \mathrm{n}=22)$. The greatest subtype diversity was found in water from drinking troughs with six subtypes (H3N2, H5N1, H5N6, H6N2, H9N2, and H11N9). However, a high percentage of the live bird market samples did not report the water source $(36.67 \%$, $\mathrm{n}=55$ ) (Figure $3 \mathrm{~A})$.

Likewise, the poultry farm samples were obtained principally from turkey barn water $(66.67 \%, \mathrm{n}=20)$ and duck's environmental water with fecal matter $(16.67 \%, \mathrm{n}=5)$; and to a lesser extent from sewage, carcass wash water, and hand-wash water $(3.33 \%, \mathrm{n}=1$ each one). The greatest subtype diversity was found in 
environmental water of duck farms with five subtypes: H3N2, H3N8, H4N9, H11N2, and H12N7. Only the poultry farm along a river in China and the free-range farm in the Netherlands did not specify the water source (Figure 3B).

The circular phylogenetic tree based on the analysis of the HA sequences detected in water samples showed that the H1 subtype was closely related to the H6 subtype, and the H12 to the H9 subtype (Figure 4). Likewise, the H7 subtype had close relation with the H10 subtype, and these were genetically close to the $\mathrm{H} 3$ and the H4 subtype.

The H3N2 HA sequence (Accession No: LC041327) detected from a live bird market in Vietnam shared 95.88\% of nucleotide sequence identity with the H3N2 HA sequence (Accession No: CY146748) from a duck farm in China. Moreover, the H3N8 HA sequence (Accession No: CY146756) isolated from a duck farm in the Dongting Lake region was grouped in a clade with H3N8 HA sequences from the Izumi plain in Japan. Meanwhile, H4 subtype evidenced two geographically separated clades, the first containing H4N6, H4N8, and H4N9 HA sequences from Asia, and the second H4N2 and H4N6 HA sequences from America. In particular, the H4N9 HA sequence (Accession No: CY146732) isolated from a duck farm close to the Dongting Lake in China was related to AIVs detected in the Izumi plain, Japan with 97.99-98.05\% nucleotide sequence identity.

All the H5N1 HA sequences were grouped together, except for one H5N1 HA sequence (Accession Ndeg: KX247930) detected in a live bird market in China, which was related to H5N6 HA sequences from a Kodai pond and the Izumi plain in Japan, as well as, from a live bird market in China with 95.95-96.34\% nucleotide sequence identity. Similarly, all the H5N8 HA sequences detected in wild bird habitats were grouped in the same clade, except the H5N8 HA sequence (Accession No EPI553362) detected in the Izumi plain in Japan which was phylogenetically related to the H5N2 HA sequences detected in turkey barns from the United States with 97.95 - $98.30 \%$ nucleotide sequence identity.

The phylogenetic analysis also showed that all the H9N2 HA sequences were grouped together in two clusters by country (China and Bangladesh) and were closely related to the H12N7 HA sequence (Accession No: CY146764) detected from a duck farm located in the Dongting Lake region in China. Finally, H11N9 HA sequences from live bird markets and the H11N2 HA sequence from a duck farm close to a wetland in China were clustered in the same clade with $95.58-95.70 \%$ nucleotide sequence identity.

\section{Discussion}

This paper describes the global AIV subtype diversity and distribution in water. It was evidenced that wild bird habitats host the highest subtype diversity reported in water samples. Wild birds of aquatic environments such as the Anseriformes and Charadriiformes constitute the major natural AIV reservoir. At least 105 wild bird species of 26 different families host LPAI, and almost all the AIV subtypes have been detected in wild aquatic bird reservoirs (Olsen et al., 2006). Wild waterfowl greatly contribute to the geographical spread of AIV subtypes between wetlands through migratory movements associated with high bird densities and increased contact rates among bird species (van Dijk et al., 2018).

Globally, a total of 112 AIV subtypes have been identified in wild birds of which 49 have been found in domestic birds (Olson et al., 2014). Only in China, twelve subtypes of HA (H1-H12) and eight subtypes of NA (N4-N9) of 21 different combinations have been identified in wild birds (Tang et al., 2020). In our analysis, we evidenced that 21 subtypes were reported from water samples, of which eleven were detected in wild bird habitats, mainly from Asian countries. Large parts of East Asia and all Southeast Asia comprise the East Asian-Australasian Flyway. This flyway supports the greatest diversity and highest number of migratory birds worldwide (Tang et al., 2020). Likewise, a large number of wild aquatic birds with a great potential to serve as carriers of AIV migrate to overwintering habitats in Asia annually (Deng et al., 2013; Khalil et al., 2020; Nakagawa et al., 2018). Nevertheless, it is well-known that geographical variations on existing surveillance efforts can also potentially influence the AIV detection and its spatial distribution (Berger et al., 2018). 
The Izumi plain and the Dongting Lake are recognized as prominent overwintering sites in Japan and China, respectively (Nakagawa et al., 2018; Zhang et al., 2011). These stopover areas for several tens of thousands of migratory birds represented the wild bird habitats with the highest number of HA sequences and the greatest subtype diversity, mainly the Izumi plain; wherein, six different subtypes (H3N8, H4N6, H4N8, H5N6, H5N8, and H6N2) were reported in water samples. Moreover, in our phylogenetic tree, most of the viruses from the Izumi plain were related to viruses from poultry farms of the Dongting Lake region in China and from turkey barns in the United States, as well as from Chinese live bird markets. Previous phylogenetic analyses of AIV strains isolated from the Izumi plain have revealed genetic reassortments between AIV from East Asian, European, African, and North American countries (Khalil et al., 2020, 2021; Nakagawa et al., 2018; Okuya et al., 2015). This trans-hemispheric genetic flow of AIV highlights the wild-domestic bird interfaces as relevant areas for influenza A virus surveillance (Prosser et al., 2013).

The Izumi plain and the Dongting Lake are also well-known for free-range farming and mixing between chickens and domestic aquatic fowl (Khalil et al., 2020). In our analyses, all the HA sequences from the subtypes H3N2, H3N8, H4N9, H11N2, and H12N7 isolated in water samples from poultry farms in China were close to aquatic environments. Domestic farming in Southern Asia commonly occurs with a lack of biosecurity measures and close contact among wild waterfowls and domestic fowls that facilitates multiple genetic reassortments (Deng et al., 2013; Zhang et al., 2011). This type of farming is widespread in low-income countries where the majority of poultry is raised under extensive conditions by family-based smallholder farms (Gilbert et al., 2015). Therefore, regions such as Eastern Europe, Central America, and sub-Saharan Africa also pose a high potential for AIV interspecies transmission; however, several high-risk areas have inadequate influenza A virus surveillance (Berger et al., 2018).

Likewise, live bird markets where different domestic and wild bird species often share the same water, food, and housing also represent an opportunity for interspecies transmission and viral genetic diversification (Zhang et al., 2011). In our analyses, most of the HA sequences were detected in water samples from live bird markets, mainly from H9N2 and H5N1 subtypes in Asia (China and Bangladesh). For more than a decade, Asian countries have undertaken numerous efforts to rapidly detect and track AIVs mainly for LPAI H9N2 and HPAI H5N1 through annual surveillance programs in poultry-related environments such as live poultry markets, poultry farms, slaughterhouses, and wild bird habitats (Rimi et al., 2019; Zhang et al., 2019). These efforts provide valuable information to inform decision-making and implement risk mitigation strategies. Unfortunately, other countries and regions do not have the same level of surveillance or do not share that data publicly, which hampers the possibility to better understand AIV transmission dynamics locally and globally (Chan et al., 2010).

The H5N1 virus continues to pose an important public health threat in East and Southeast Asian countries and has become endemic in domestic poultry in these countries (Rimi et al., 2019). Although HPAI H5N1 has also caused many outbreaks with severe illness in poultry from Europe and Africa (Chowdhury et al., 2019), in our analyses HPAI H5N1 viruses were only reported in water samples from Asian countries. Similarly, in our study LPAI H9N2 viruses were isolated solely in water samples from Asia. Nevertheless, LPAI H9N2 avian influenza viruses have widespread in domestic poultry worldwide (Peacock et al., 2019).

Multiple AIV subtypes have been detected from poultry and wild birds in Africa, Australia, Europe, and America (Alexander, 2007; Araujo et al., 2018; Brown, 2010; Grillo et al., 2015; Hansbro et al., 2010; Jimenez-Bluhm et al., 2018; Senne, 2007). However, our global analysis of AIV evidenced a lack of sequences isolated from environmental water in Africa, Oceania, and South America, as well as, in Europe. Data on influenza in tropical countries remain scarce compared with temperate countries (Moura, 2010). Likewise, according to Moura (2010), the emergence of AIV strains in Asia may occur and be detected approximately 6 to 9 months earlier than in Oceania, North America, and Europe, and 12 to 18 months earlier than in South America.

It is also recognized that the prevalence and distribution of avian influenza in most tropical countries are mostly unknown as a result of the lack of a rigorous surveillance system (Yazdanbakhsh \& Kremsner, 2009). Since the H1N1 pandemic, it has been highlighted a considerable number of shortcomings on global epidemi- 
ological surveillance. The absence of routine AIV monitoring has resulted in substantial information gaps in large areas of the world, mainly from less-resourced countries (Briand et al., 2011).

Environmental sampling has been effectively used for AIV surveillance since the 1970s. Nevertheless, the methods and protocols are not completely standardized, as well as, an international guideline about data management is absent (Hood et al., 2020). This was one of the most noticeable limitations in our analyses since several AIV no specified the type of environment sampled (i.e., feces, water, air, mud, or surface swabs), and thus, those HA sequences were excluded. Likewise, we only analyzed the sequences with complete subtypes reported in the four recognized electronic databases. In the case of Europe, there were reported 83 sequences of $\mathrm{H} 1$ genes from ice and water in high-latitude lakes visited by large numbers of migratory birds in Siberia. Nevertheless, those sequences did not meet the inclusion criteria. We also found several geographical regions with missing subtype data. Therefore, the AIV subtypes described in this work may not be the exact reflection of the global subtype diversity, but it highlights the potential value of using this information to better understand AIV local and global transmission dynamics. Our work also highlights the need to improve surveillance efforts in many regions as well as to advance towards more unified data collection and sharing standards to improve influenza A virus surveillance and better prevent future potential pandemics.

In conclusion, this descriptive and phylogenetic analysis of AIVs isolated in water samples from sites at high risk for influenza outbreaks, such as live bird markets, poultry farms, and wild bird habitats is valuable to provide an overview and baseline information of the current data on global AIV diversity and distribution since 2003. However, this study highlights the need to continuing generating, expanding, and sharing precise and detailed environmental data from surveillance systems to allow a better understanding of the ecology and epidemiology of AIV, especially from low- and middle-income countries.

\section{Acknowledgements}

This research belongs to the PAPIIT-DGAPA project Ndeg IN222119. Jessica Mateus-Anzola is a doctoral student of the 'Posgrado en Ciencias de la Produccion y de la Salud Animal', Universidad Nacional Autonoma de Mexico (UNAM). The authors are grateful to the Mexican National Council for Science and Technology for postgraduate fellowship (CONACyT, ID 785318).

\section{Conflict of Interest}

The authors declare that they have no conflict of interest.

\section{Data Availability Statement}

Data supporting the findings of this study are included in the supporting file. The HA sequences of avian influenza viruses are available in the public-access databases mentioned in this manuscript.

\section{Ethics Statement}

No ethical approval was required as this is an article that analyses public-access data.

\section{References}

Achenbach, J. E., \& Bowen, R. A. (2011). Transmission of Avian Influenza A Viruses among Species in an Artificial Barnyard. PLoS ONE , 6 (3), e17643. https://doi.org/10.1371/journal.pone.0017643

Alexander, D. J. (2007). Summary of Avian Influenza Activity in Europe, Asia, Africa, and Australasia, 2002-2006. Avian Diseases ,51 (s1), 161-166. https://doi.org/10.1637/7602-041306R.1

Araujo, J., Petry, M. V., Fabrizio, T., Walker, D., Ometto, T., Thomazelli, L. M., Scherer, A. L., Serafini, P. P., Neto, I. S., Krauss, S., Webster, R. G., Webby, R. J., \& Durigon, E. L. (2018). Migratory birds in southern Brazil are a source of multiple avian influenza virus subtypes. Influenza and Other Respiratory Viruses , 12 (2), 220-231. https://doi.org/10.1111/irv.12519 
Berger, K. A., Pigott, D. M., Tomlinson, F., Godding, D., Maurer-Stroh, S., Taye, B., Sirota, F. L., Han, A., Lee, R. T. C., Gunalan, V., Eisenhaber, F., Hay, S. I., \& Russell, C. A. (2018). The Geographic Variation of Surveillance and Zoonotic Spillover Potential of Influenza Viruses in Domestic Poultry and Swine. Open Forum Infectious Diseases , 5 (12), ofy318. https://doi.org/10.1093/ofid/ofy318

Briand, S., Mounts, A., \& Chamberland, M. (2011). Challenges of global surveillance during an influenza pandemic. Public Health ,125 (5), 247-256. https://doi.org/10.1016/j.puhe.2010.12.007

Brown, I. H. (2010). Summary of Avian Influenza Activity in Europe, Asia, and Africa, 2006-2009. Avian Diseases , 54 (s1), 187-193. https://doi.org/10.1637/8949-053109-Reg.1

Brown, J. D., Swayne, D. E., Cooper, R. J., Burns, R. E., \& Stallknecht, D. E. (2007). Persistence of H5 and H7 Avian Influenza Viruses in Water. Avian Diseases , 51 (s1), 285-289. https://doi.org/10.1637/7636042806R.1

Cappelle, J., Gaidet, N., Iverson, S. A., Takekawa, J. Y., Newman, S. H., Fofana, B., \& Gilbert, M. (2011). Characterizing the interface between wild ducks and poultry to evaluate the potential of transmission of avian pathogens. International Journal of Health Geographics ,10 (1), 60. https://doi.org/10.1186/1476072X-10-60

Chan, J., Holmes, A., \& Rabadan, R. (2010). Network Analysis of Global Influenza Spread. PLoS Computational Biology , 6 (11), e1001005. https://doi.org/10.1371/journal.pcbi.1001005

Chowdhury, Hossain, Ghosh, Ghosh, Hossain, Beard, Rahman, \& Rahman. (2019). The Pattern of Highly Pathogenic Avian Influenza H5N1 Outbreaks in South Asia. Tropical Medicine and Infectious Disease ,4 (4), 138. https://doi.org/10.3390/tropicalmed4040138

Deng, G., Tan, D., Shi, J., Cui, P., Jiang, Y., Liu, L., Tian, G., Kawaoka, Y., Li, C., \& Chen, H. (2013). Complex Reassortment of Multiple Subtypes of Avian Influenza Viruses in Domestic Ducks at the Dongting Lake Region of China. Journal of Virology ,87 (17), 9452-9462. https://doi.org/10.1128/JVI.00776-13

Dovas, C. I., Papanastassopoulou, M., Georgiadis, M. P., Chatzinasiou, E., Maliogka, V. I., \& Georgiades, G. K. (2010). Detection and Quantification of Infectious Avian Influenza A (H5N1) Virus in Environmental Water by Using Real-Time Reverse Transcription-PCR.Applied and Environmental Microbiology , 76 (7), 2165-2174. https://doi.org/10.1128/AEM.01929-09

Edgar, R. C. (2004). MUSCLE: a multiple sequence alignment method with reduced time and space complexity. BMC Bioinformatics ,5 (1), 113. https://doi.org/10.1186/1471-2105-5-113

Gilbert, M., Conchedda, G., Van Boeckel, T. P., Cinardi, G., Linard, C., Nicolas, G., Thanapongtharm, W., D'Aietti, L., Wint, W., Newman, S. H., \& Robinson, T. P. (2015). Income Disparities and the Global Distribution of Intensively Farmed Chicken and Pigs. PLOS ONE ,10 (7), e0133381. https://doi.org/10.1371/journal.pone.0133381

Grillo, V., Arzey, K., Hansbro, P., Hurt, A., Warner, S., Bergfeld, J., Burgess, G., Cookson, B., Dickason, C., Ferenczi, M., Hollingsworth, T., Hoque, M., Jackson, R., Klaassen, M., Kirkland, P., Kung, N., Lisovski, S., O'Dea, M., O'Riley, K., ... Post, L. (2015). Avian influenza in Australia: A summary of 5 years of wild bird surveillance.Australian Veterinary Journal , 93 (11), 387-393. https://doi.org/10.1111/avj.12379

Hansbro, P. M., Warner, S., Tracey, J. P., Arzey, K. E., Selleck, P., O'Riley, K., Beckett, E. L., Bunn, C., Kirkland, P. D., Vijaykrishna, D., Olsen, B., \& Hurt, A. C. (2010). Surveillance and Analysis of Avian Influenza Viruses, Australia. Emerging Infectious Diseases ,16 (12), 1896-1904. https://doi.org/10.3201/eid1612.100776

Harris, K. A., Freidl, G. S., Munoz, O. S., von Dobschuetz, S., De Nardi, M., Wieland, B., Koopmans, M. P. G., Stark, K. D. C., van Reeth, K., Dauphin, G., Meijer, A., de Bruin, E., Capua, I., Hill, A. A., Kosmider, R., Banks, J., Stevens, K., van der Werf, S., Enouf, V., ... Breed, A. C. (2017). Epidemiolog- 
ical Risk Factors for Animal Influenza A Viruses Overcoming Species Barriers. EcoHealth ,14 , 342-360. https://doi.org/10.1007/s10393-017-1244-y

Hood, G., Roche, X., Brioudes, A., von Dobschuetz, S., Fasina, F. O., Kalpravidh, W., Makonnen, Y., Lubroth, J., \& Sims, L. (2020). A literature review of the use of environmental sampling in the surveillance of avian influenza viruses. Transboundary and Emerging Diseases , 00 , 1-17. https://doi.org/10.1111/tbed.13633

Jimenez-Bluhm, P., Karlsson, E. A., Freiden, P., Sharp, B., Di Pillo, F., Osorio, J. E., Hamilton-West, C., \& Schultz-Cherry, S. (2018). Wild birds in Chile Harbor diverse avian influenza A viruses. Emerging Microbes E Infections , 7 (1), 1-4. https://doi.org/10.1038/s41426-018-0046-9

Karasin, A. I., Brown, I. H., Carman, S., \& Olsen, C. W. (2000). Isolation and Characterization of H4N6 Avian Influenza Viruses from Pigs with Pneumonia in Canada. Journal of Virology , 74 (19), 9322-9327. https://doi.org/10.1128/JVI.74.19.9322-9327.2000

Keeler, S. P., Dalton, M. S., Cressler, A. M., Berghaus, R. D., \& Stallknecht, D. E. (2014). Abiotic Factors Affecting the Persistence of Avian Influenza Virus in Surface Waters of Waterfowl Habitats.Applied and Environmental Microbiology , 80 (9), 2910-2917. https://doi.org/10.1128/AEM.03790-13

Khalil, A. M., Fujimoto, Y., Kojima, I., Esaki, M., Ri, K., Masatani, T., Matsui, T., \& Ozawa, M. (2021). Genetic Characterization of H5N8 Highly Pathogenic Avian Influenza Viruses Isolated from Falcated Ducks and Environmental Water in Japan in November 2020. Pathogens ,10 (2), 171. https://doi.org/10.3390/pathogens10020171

Khalil, A. M., Nishi, N., Kojima, I., Fukunaga, W., Kuwahara, M., Masatani, T., Matsui, T., \& Ozawa, M. (2020). Transition in genetic constellations of H3N8 and H4N6 low-pathogenic avian influenza viruses isolated from an overwintering site in Japan throughout different winter seasons. Archives of Virology , 165 (3), 643-659. https://doi.org/10.1007/s00705-019-04519-z

Khan, S. U., Gurley, E. S., Gerloff, N., Rahman, M. Z., Simpson, N., Rahman, M., Haider, N., Chowdhury, S., Balish, A., Zaman, R. U., Nasreen, S., Chandra Das, B., Azziz-Baumgartner, E., Sturm-Ramirez, K., Davis, C. T., Donis, R. O., \& Luby, S. P. (2018). Avian influenza surveillance in domestic waterfowl and environment of live bird markets in Bangladesh, 2007-2012. Scientific Reports , 8 (1), 9396. https://doi.org/10.1038/s41598-018-27515-w

Kumar, S., Stecher, G., Li, M., Knyaz, C., \& Tamura, K. (2018). MEGA X: Molecular Evolutionary Genetics Analysis across Computing Platforms.Molecular Biology and Evolution , 35 (6), 1547-1549. https://doi.org/10.1093/molbev/msy096

Lebarbenchon, C., Yang, M., Keeler, S. P., Ramakrishnan, M. A., Brown, J. D., Stallknecht, D. E., \& Sreevatsan, S. (2011). Viral Replication, Persistence in Water and Genetic Characterization of Two Influenza A Viruses Isolated from Surface Lake Water. PLoS ONE , 6 (10), e26566. https://doi.org/10.1371/journal.pone.0026566

Leung, Y. H. C., Zhang, L.-J., Chow, C.-K., Tsang, C.-L., Ng, C.-F., Wong, C.-K., Guan, Y., \& Peiris, J. S. M. (2007). Poultry Drinking Water Used for Avian Influenza Surveillance. Emerging Infectious Diseases , 13 (9), 1380-1382. https://doi.org/10.3201/eid1309.070517

Lickfett, T. M., Clark, E., Gehring, T. M., \& Alm, E. W. (2018). Detection of Influenza A viruses at migratory bird stopover sites in Michigan, USA. Infection Ecology \& Epidemiology , 8 (1), 1474709. https://doi.org/10.1080/20008686.2018.1474709

Ma, W., Vincent, A. L., Gramer, M. R., Brockwell, C. B., Lager, K. M., Janke, B. H., Gauger, P. C., Patnayak, D. P., Webby, R. J., \& Richt, J. A. (2007). Identification of H2N3 influenza A viruses from swine in the United States. Proceedings of the National Academy of Sciences ,104 (52), 20949-20954. https://doi.org/10.1073/pnas.0710286104 
Machalaba, C. C., Elwood, S. E., Forcella, S., Smith, K. M., Hamilton, K., Jebara, K. B., Swayne, D. E., Webby, R. J., Mumford, E., Mazet, J. A. K., Gaidet, N., Daszak, P., \& Karesh, W. B. (2015). Global Avian Influenza Surveillance in Wild Birds: A Strategy to Capture Viral Diversity. Emerging Infectious Diseases , 21 (4), e141415. https://doi.org/10.3201/eid2104.141415

Montalvo-Corral, M., \& Hernandez, J. (2010). Genetic analysis of avian influenza virus from migratory waterfowl in Mexico. Archives of Virology , 155 (1), 97-101. https://doi.org/10.1007/s00705-009-0554-y

Moura, F. E. (2010). Influenza in the tropics: Current Opinion in Infectious Diseases , 23 (5), 415-420. https://doi.org/10.1097/QCO.0b013e32833cc955

Nakagawa, H., Okuya, K., Kawabata, T., Matsuu, A., Takase, K., Kuwahara, M., Toda, S., \& Ozawa, M. (2018). Genetic characterization of low-pathogenic avian influenza viruses isolated on the Izumi plain in Japan: Possible association of dynamic movements of wild birds with AIV evolution. Archives of Virology , 163 (4), 911-923. https://doi.org/10.1007/s00705-017-3698-1

Numberger, D., Dreier, C., Vullioud, C., Gabriel, G., Greenwood, A. D., \& Grossart, H.-P. (2019). Recovery of influenza A viruses from lake water and sediments by experimental inoculation. PLOS ONE ,14 (5), e0216880. https://doi.org/10.1371/journal.pone.0216880

Okuya, K., Kawabata, T., Nagano, K., Tsukiyama-Kohara, K., Kusumoto, I., Takase, K., \& Ozawa, M. (2015). Isolation and characterization of influenza A viruses from environmental water at an overwintering site of migratory birds in Japan. Archives of Virology , 160 (12), 3037-3052. https://doi.org/10.1007/s00705015-2610-0

Olsen, B., Munster, V. J., Wallensten, A., Waldenstrom, J., Osterhaus, A. D. M. E., \& Fouchier, R. A. M. (2006). Global Patterns of Influenza A Virus in Wild Birds. Science , 312 (5772), 384-388. https://doi.org/10.1126/science.1122438

Olson, S. H., Parmley, J., Soos, C., Gilbert, M., Latorre-Margalef, N., Hall, J. S., Hansbro, P. M., Leighton, F., Munster, V., \& Joly, D. (2014). Sampling Strategies and Biodiversity of Influenza A Subtypes in Wild Birds. PLoS ONE , 9 (3), e90826. https://doi.org/10.1371/journal.pone.0090826

Peacock, T. P., James, J., Sealy, J. E., \& Iqbal, M. (2019). A Global Perspective on H9N2 Avian Influenza Virus. Viruses, 11 (7), 620. https://doi.org/10.3390/v11070620

Prosser, D. J., Hungerford, L. L., Erwin, R. M., Ottinger, M. A., Takekawa, J. Y., \& Ellis, E. C. (2013). Mapping Avian Influenza Transmission Risk at the Interface of Domestic Poultry and Wild Birds.Frontiers in Public Health , 1 , 28. https://doi.org/10.3389/fpubh.2013.00028

Rambaut, A. (2018). FigTree v1.4.4. Institute of Evolutionary Biology, University of Edinburgh, Edinburgh. http://tree.bio.ed.ac.uk/software/figtree/

Rimi, Hassan, Chowdhury, Rahman, Sultana, Biswas, Debnath, Islam, \& Ross. (2019). A Decade of Avian Influenza in Bangladesh: Where Are We Now? Tropical Medicine and Infectious Disease , 4 (3), 119. https://doi.org/10.3390/tropicalmed4030119

Roche, B., Lebarbenchon, C., Gauthier-Clerc, M., Chang, C.-M., Thomas, F., Renaud, F., van der Werf, S., \& Guegan, J.-F. (2009). Water-borne transmission drives avian influenza dynamics in wild birds: The case of the 2005-2006 epidemics in the Camargue area. Infection, Genetics and Evolution, 9 (5), 800-805. https://doi.org/10.1016/j.meegid.2009.04.009

Senne, D. A. (2007). Avian Influenza in North and South America, 2002-2005. Avian Diseases , 51 (s1), 167-173. https://doi.org/10.1637/7621-042606R1.1

Tang, L., Tang, W., Li, X., Hu, C., Wu, D., Wang, T., \& He, G. (2020). Avian Influenza Virus Prevalence and Subtype Diversity in Wild Birds in Shanghai, China, 2016-2018. Viruses , 12 (9), 1031. https://doi.org/10.3390/v12091031 
van Dijk, J. G., Verhagen, J. H., Wille, M., \& Waldenstrom, J. (2018). Host and virus ecology as determinants of influenza A virus transmission in wild birds. Current Opinion in Virology , 28 , 26-36. https://doi.org/10.1016/j.coviro.2017.10.006

Vergne, T., Meyer, A., Long, P. T., Elkholly, D. A., Inui, K., Padungtod, P., Newman, S. H., Fournie, G., \& Pfeiffer, D. U. (2019). Optimising the detectability of H5N1 and H5N6 highly pathogenic avian influenza viruses in Vietnamese live-bird markets. Scientific Reports , 9 (1), 1031. https://doi.org/10.1038/s41598018-37616-1

Wickham, H. (2016). ggplot2: Elegant Graphics for Data Analysis.Springer-Verlag New York. https://ggplot2.tidyverse.org.

Yazdanbakhsh, M., \& Kremsner, P. G. (2009). Influenza in Africa.PLoS Medicine , 6 (12), e1000182. https://doi.org/10.1371/journal.pmed.1000182

Zhang, H., Xu, B., Chen, Q., Chen, J., \& Chen, Z. (2011). Characterization of an H10N8 influenza virus isolated from Dongting lake wetland. Virology Journal , 8 (1), 42. https://doi.org/10.1186/1743-422X-8-42

Zhang, H., Xu, B., Chen, Q., \& Chen, Z. (2011). Characterization of H9N2 influenza viruses isolated from Dongting Lake wetland in 2007. Archives of Virology , 156 (1), 95-105. https://doi.org/10.1007/s00705-0100836-4

Zhang, Y., Dong, J., Bo, H., Dong, L., Zou, S., Li, X., Shu, Y., \& Wang, D. (2019). Genetic and biological characteristics of avian influenza virus subtype $\mathrm{H} 1 \mathrm{~N} 8$ in environments related to live poultry markets in China. BMC Infectious Diseases , 19 (1), 458. https://doi.org/10.1186/s12879-019-4079-z

Figure legend

Figure 1. AIV subtypes detected in water samples by country. A total of 21 subtypes from nine countries were isolated between $2003-2020$. The H9N2 subtype was the most reported $(40.17 \%, \mathrm{n}=94)$, followed by the H5N1 $(23.07 \%, \mathrm{n}=54)$ and the H5N6 $(8.97 \%, \mathrm{n}=21)$. The world map shows the number of AIV subtypes reported by country; red, orange, and yellow areas indicate high, medium, and low subtype diversity, respectively. The map was created online athttps://mapchart.net.

Figure 2. The number of AIV sequences detected in water samples by sampling site. A) live bird markets (LBM), B) wild bird habitats (WBH), C) poultry farms (PF), D) unknown (UNK) sampling site. Red, orange, and yellow areas in the map indicate high, medium, and low number of AIV sequences, respectively. The pie charts show the proportion of each subtype by country in each sampling site. The bar chart presents the number of AIV sequences by country and sampling site. The map was created online athttps://mapchart.net.

Figure 3. Number of AIV sequences by water source and subtype.A) Live bird market, B) Poultry farm. Carcass-wash water (CWW), duck's environmental water (DEW), drinking water trough (DW), handwash water $(\mathrm{HWW})$, poultry cage water $(\mathrm{PCW})$, sewage $(\mathrm{SW})$, turkey barn water (TBW), and unknown (UNK). The highest subtype diversity was found in DW from live bird markets, and in DEW from a poultry farm.

Figure 4. Circular phylogenetic tree of hemagglutinin (HA) gene sequences of AIV detected in

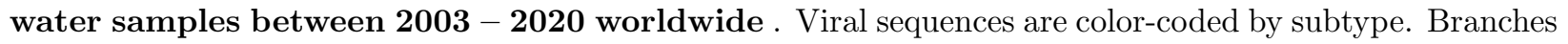
are color-coded by sampling site (red= live bird market $(\mathrm{LBM})$, blue $=\mathrm{WBH}$ (wild bird habitat), purple $=$ poultry farm $(\mathrm{PF})$, and black= unknown $(\mathrm{UNK}))$. The tree was generated with the Maximum-likelihood method using MEGA X and edited with FigTree v1.4.4 software.

\section{Supporting information}

Table S1. Accession numbers of AIV hemagglutinin (HA) gene sequences detected in water samples from $2003-2020$. 

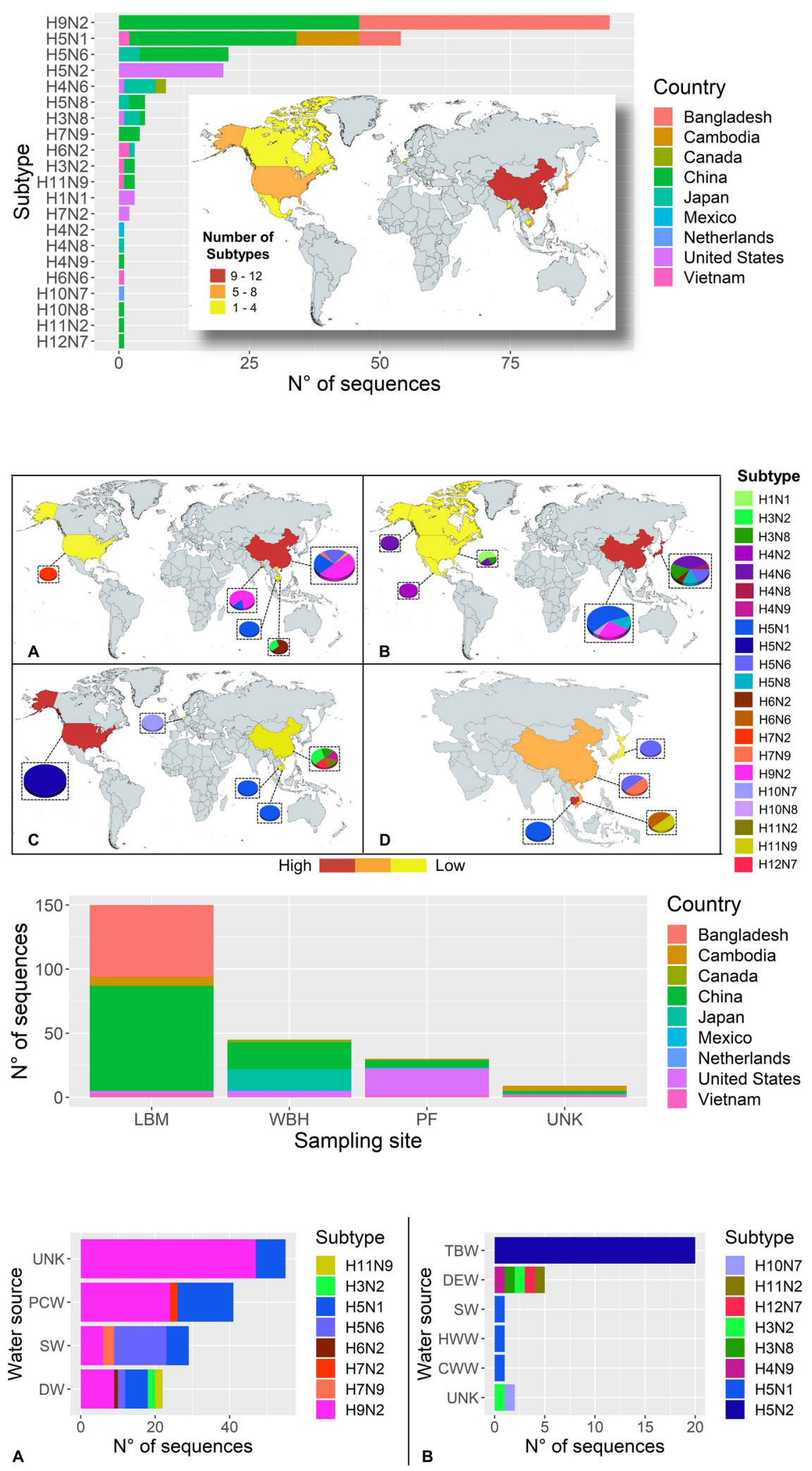


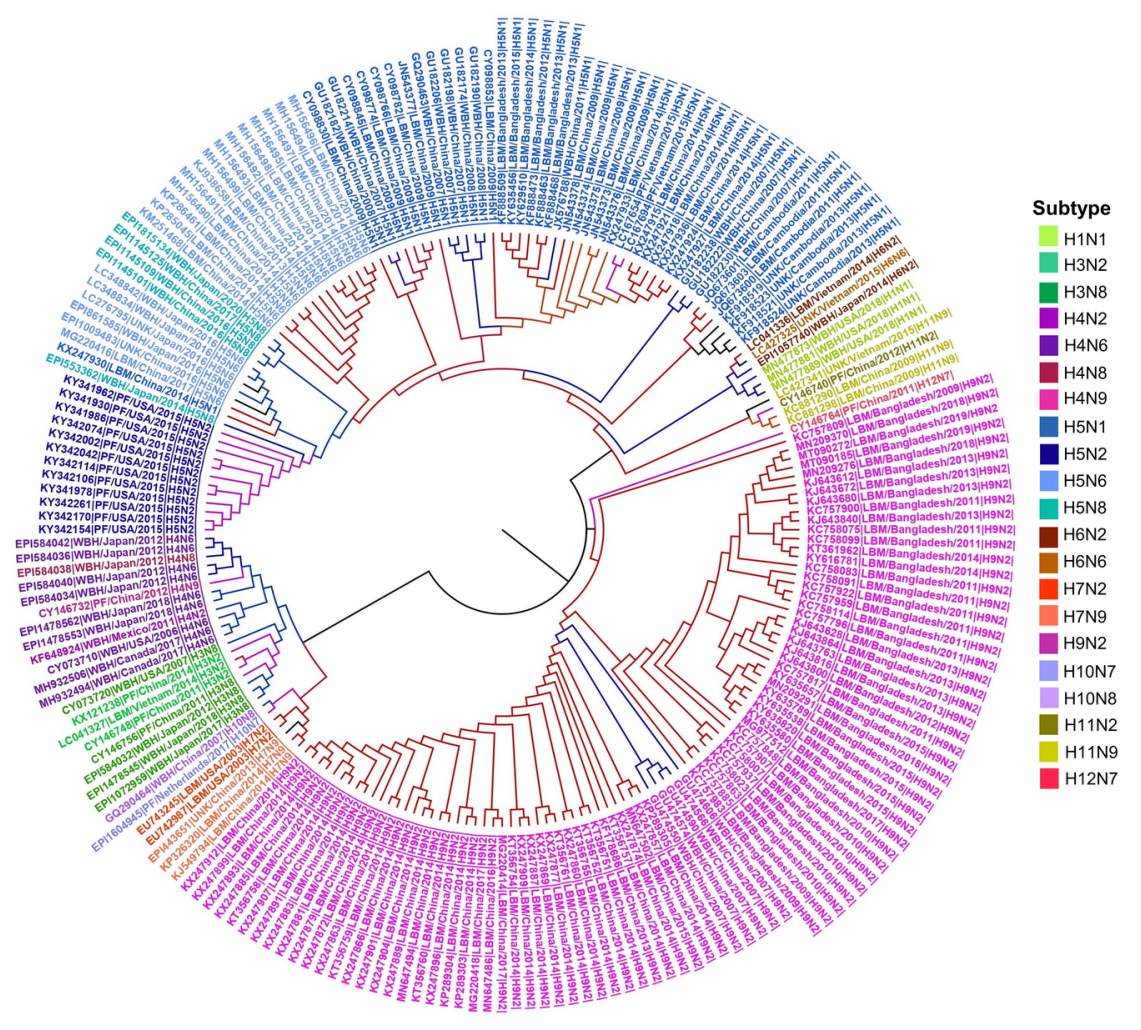

